\title{
Band lineup of lattice mismatched InSe/GaSe quantum well structures prepared by van der Waals epitaxy: Absence of interfacial dipoles
}

Cite as: Journal of Applied Physics 80, 3817 (1996); https://doi.org/10.1063/1.363335

Submitted: 01 April 1996 • Accepted: 20 June 1996 • Published Online: 04 June 1998

O. Lang, A. Klein, C. Pettenkofer, et al.

ARTICLES YOU MAY BE INTERESTED IN

Thin film growth and band lineup of $\operatorname{In}_{2} \mathrm{O}_{3}$ on the layered semiconductor InSe

Journal of Applied Physics 86, 5687 (1999); https://doi.org/10.1063/1.371579

Band lineup of layered semiconductor heterointerfaces prepared by van der Waals epitaxy:

Charge transfer correction term for the electron affinity rule

Journal of Applied Physics 85, 2732 (1999); https://doi.org/10.1063/1.369590

Crystal structure and optical performance in bulk $\gamma$-InSe single crystals

AIP Advances 9, 025013 (2019); https://doi.org/10.1063/1.5086492

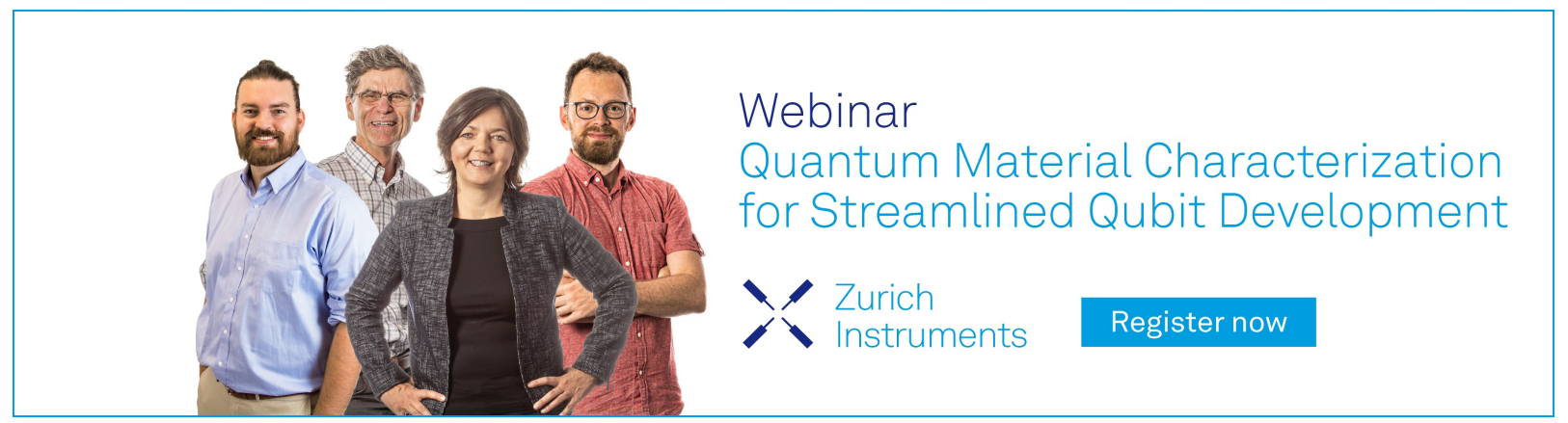




\title{
Band lineup of lattice mismatched InSe/GaSe quantum well structures prepared by van der Waals epitaxy: Absence of interfacial dipoles
}

\author{
O. Lang, A. Klein, C. Pettenkofer, and W. Jaegermann ${ }^{\text {a) }}$ \\ Hahn-Meitner-Institut, Abteilung CG, Glienicker Str. 100, 14109 Berlin, Germany
}

\author{
A. Chevy \\ Physique de Milieux Condensés, Université Pierre et Marie Curie, 75252 Paris Cedex 05, France
}

(Received 1 April 1996; accepted for publication 20 June 1996)

\begin{abstract}
Epitaxial growth of the strongly lattice mismatched (6.5\%) layered chalcogenides InSe and GaSe on each other is obtained with the concept of van der Waals epitaxy as proven by low-energy electron diffraction and scanning tunnel microscope. InSe/GaSe/InSe and $\mathrm{GaSe} / \mathrm{InSe} / \mathrm{GaSe}$ quantum well structures were prepared by molecular beam epitaxy and their interface properties were characterized by soft x-ray photoelectron spectroscopy. Valence and conduction band offsets are determined to be 0.1 and $0.9 \mathrm{eV}$, respectively, and do not depend on deposition sequence (commutativity). As determined from the measured work functions the interface dipole is $0.05 \mathrm{eV}$; the band lineup between the two materials is correctly predicted by the Anderson model (electron affinity rule). (C) 1996 American Institute of Physics. [S0021-8979(96)09018-4]
\end{abstract}

\section{INTRODUCTION}

Band lineup of semiconductor heterointerfaces is the most dominant parameter which determines the optoelectronic properties of heterojunctions. But in spite of numerous experimental and theoretical studies (compare, e.g., Ref. 1 and references therein) the underlying principles which govern the alignment of the bands are still a matter of debate. Especially the relative importance of bulk versus surface or interface properties is still not settled. In the original proposal of Anderson ${ }^{2}$ the band alignment is determined from the electron affinities of the semiconductors. The electron affinity of a solid is strongly influenced by surface dipoles which are given by the local atomic structure and charge distribution at the surface. Band alignment predictions from the electron affinity rule (EAR) will thus only be valid if the changes of these dipoles at the interface are equal for both semiconductors. However, this is generally not to be expected and it is not surprising that the experimentally determined band alignments for most three-dimensional semiconductor heterointerfaces do not follow the EAR. ${ }^{1}$

In the tetrahedrally bound three-dimensional semiconductors the electronic structure at the interface (surface) is, in general, different from that in the bulk. The new electronic interface states are considered to depend on the bulk band structure. The band alignment of the semiconductors is then governed by the so-called charge neutrality level of these interface states which are often situated close to midgap. ${ }^{3-5}$ The most accepted theory by Tersoff for nonreacting interfaces ${ }^{3}$ predicts virtual gap states (VIGSs) at the heterointerface which are derived by wave functions of both semiconductors. The same charge neutrality levels also determine the barrier heights at semiconductor/metal interfaces. ${ }^{6}$

However, for the three-dimensional semiconductors the atomic structure at the interface is usually different from that in the bulk. It can even change depending on the preparation and/or orientation of the interface. In fact it has been shown

${ }^{a)}$ Corresponding author; Electronic mail: jaegermann@hmi.de. in very recent experimental and theoretical studies that band offsets can change appreciably with interfacial atomic composition. ${ }^{7}$ These results show that the band discontinuities cannot be predicted from bulk properties alone as is done in most theories subsequent to the EAR which align the charge neutrality levels.

Different situation is given for heterointerfaces between materials with a quasi two-dimensional structure, e.g., the layered chalcogenide semiconductors. These layered compounds are characterized by a strongly anisotropic structure with covalently bound $X-M-X \quad$ (e.g., $\mathrm{MoS}_{2}$ ) or $X-M-M-X$ (e.g., InSe, GaSe) layers attached to each other in the third dimension by weak interactions. The bonding between the layers is usually referred to as van der Waals like. The (0001) van der Waals cleavage planes contain a hexagonally closed packed chalcogen layer without dangling bonds.

It has been shown recently that such surfaces also allow the epitaxial growth of semiconductor overlayers of high crystalline quality despite strong lattice mismatch [van der Waals epitaxy, (VDWE)]. ${ }^{8,9}$ The interfaces are found to be nonreactive and therefore considered as atomically abrupt. Due to the weak bonding between the layers there is almost no charge accumulation in the van der Waals gap as shown by recent band structure calculations. ${ }^{10}$ Negligible charge redistribution could then be expected when changing from a homointerface (inner surface) either to the surface (vacuum) or to a heterointerface. If this is true, the band lineup should be predicted correctly by the EAR.

In this article we report on the electronic properties of InSe/GaSe/InSe and $\mathrm{GaSe} / \mathrm{InSe} / \mathrm{GaSe}$ quantum well structures prepared by VDWE. The structures were characterized by in situ electron diffraction (LEED) and soft X-ray photoelectron spectroscopy (SXPS). The VDWE of InSe and GaSe on different layered substrates has been proven recently. ${ }^{11-15}$ Also the heterointerface InSe/GaSe has already been investigated by XPS, ${ }^{14,15}$ but the reported band discontinuities differ from each other. 

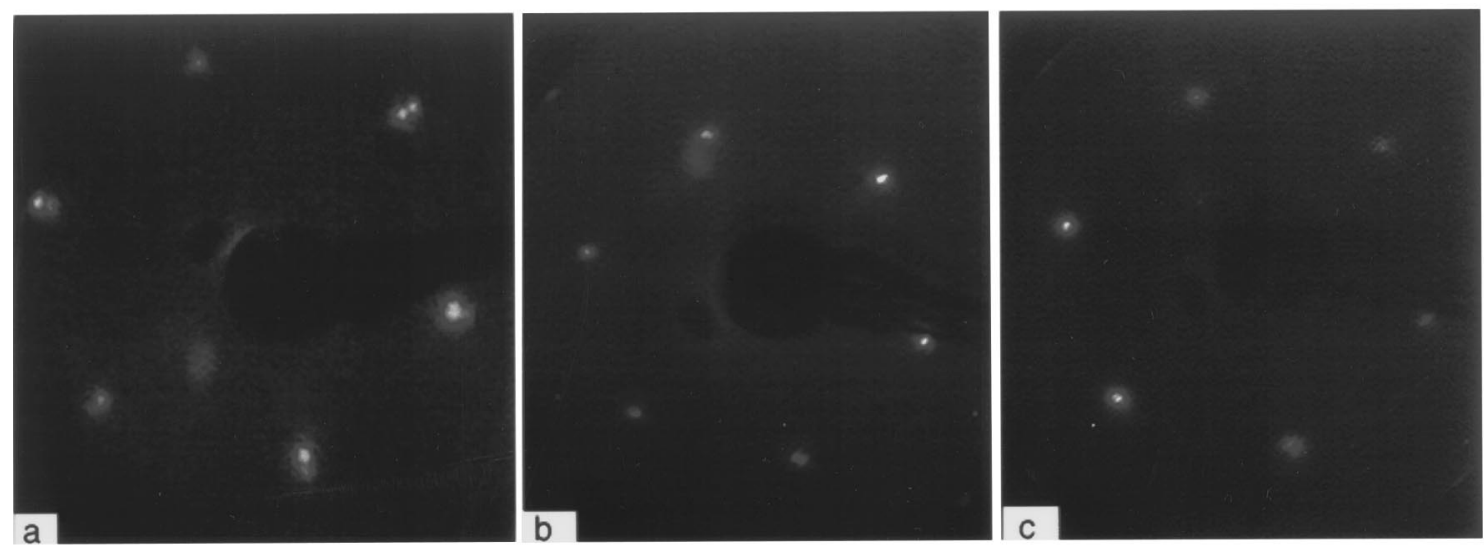

FIG. 1. LEED patterns taken from different InSe and GaSe films. (a) $\Theta=20 \AA$ InSe on GaSe $\left(E_{\text {kin }}=46\right.$ eV); (b) $\Theta=90 \AA$ InSe on GaSe $\left(E_{\text {kin }}=67\right.$ eV); (c) $\Theta=190 \AA$ GaSe on $\operatorname{InSe}\left(E_{\text {kin }}=67 \mathrm{eV}\right)$.

\section{EXPERIMENT}

All molecular beam epitaxy (MBE) experiments were performed in a home made growth chamber connected to a surface analysis system equipped with a VG ADES 500 angle resolving electron spectrometer and LEED. Valence and core-level photoelectron spectroscopy were taken with synchrotron radiation in the energy range $h \nu=20-120 \mathrm{eV}$ at the TGM 7 beamline of the BESSY synchrotron. As substrates single crystals of GaSe and InSe prepared by standard bridgman technique are used. ${ }^{16}$ The samples were cleaved in air and subsequently heated in UHV at $400{ }^{\circ} \mathrm{C}$ for $1 \mathrm{~h}$ to give clean (0001) surfaces. The growth chamber was equipped with a heatable sample manipulator $\left(T_{\max }=900 \mathrm{~K}\right)$. The sample temperature was controlled by a thermocouple directly attached to the sample holder. GaSe and InSe overlayers were grown by evaporation of crushed GaSe and InSe single crystals from home made MBE Knudsen cells. A quartz microbalance was used to determine the deposition rates. The measurements were in some cases supplemented ex situ by scanning tunneling microscopy (STM). ${ }^{17}$

\section{RESULTS AND DISCUSSIONS}

Some experiment results on the growth of VDWE films of InSe and GaSe have already been described elsewhere. ${ }^{11,12,17}$ Examples of LEED results of InSe films deposited on GaSe (0001) substrates and vice versa are shown in Fig. 1. The LEED patterns shown in Figs. 1(a) and 1(b) were taken for nominal InSe thicknesses of $\Theta=20 \AA$ and $\Theta=90 \AA$. Figure 1(c) shows the LEED pattern of a GaSe film of $\Theta=190 \AA$ nominal thickness. The GaSe overlayer was grown at a sample temperature $T_{s}=420^{\circ} \mathrm{C}$. Growth of InSe overlayers was done at sample temperatures $T_{s}=300-370{ }^{\circ} \mathrm{C}$. The overlayers clearly exhibit hexagonal diffraction patterns, indicating single crystalline epitaxial overlayer growth. For thin films the hexagonal diffraction patterns from the substrate and film are both visible (see also Refs. 11, 12, and 17). Their azimuths are well aligned. The angular ratios are determined to be $6.7 \%$ for InSe on $\mathrm{GaSe}$ and $6.25 \%$ for $\mathrm{GaSe}$ on InSe. These match closely to the inverse ratio of the bulk lattice constants of the two materials $\left[a_{0}(\mathrm{GaSe})=3.75 \AA ; a_{0}(\mathrm{InSe})=4.0 \AA\right]$. It should be men- tioned that the occurrence of double diffraction spots in the low coverage regime is only possible for incomplete coverages. The spots from the substrate result from uncovered parts of the surface while those from the overlayer are not influenced by the substrate. The occurrence of small holes in the overlayer is also evident from STM pictures. ${ }^{12,17}$

In Fig. 2, SXPS results of the In4d and Ga3d core levels obtained for the deposition sequences $\mathrm{GaSe} / \mathrm{InSe} / \mathrm{GaSe}$ [Fig. 2(a)] and InSe/GaSe/InSe [Fig. 2(b)], respectively, are summarized. In both cases the effect of intermediate annealing steps is also shown. The substrate and overlayer emission lines remain very sharp during deposition indicating the formation of a nonreactive and atomically abrupt interface. Only small band bending (InSe on GaSe: $\triangle B E=0.37 \mathrm{eV}$; GaSe on InSe: $\triangle B E=0.2 \mathrm{eV}$ ) is observed after the initial deposition steps. The strong attenuation of the substrate emission line with overlayer thickness indicates mostly layer-by-layer growth (Frank-van der Merwe growth mode). After the annealing steps, which are introduced to improve the crystalline quality of the film, the substrate emissions again increase in intensity. This is explained by the formation of islands.

Valence band spectra taken with excitation energy $h \nu$ $=40 \mathrm{eV}$ for both deposition sequences are shown in Fig. 3 . Spectra from cleaved single crystal surfaces of GaSe and InSe are given at the bottom. Their spectral features are mainly reproduced in the spectra taken from the thicker films. We take this as further evidence for epitaxial growth and nonreactive interface formation. Remaining differences between film and single crystal spectra are explained by small deviations from normal emission geometry, as we have checked by rotating the analyzer. The spectra change appreciably even for $\pm 3^{\circ}$ off normal emission due to energy band dispersion.

Valence band offset of the interfaces is determined from the relative position of core level lines of the heterointerfaces when the binding energy of the lines are known with respect to the valence band maximum (VBM). Following the procedure described by Waldrop and Grant ${ }^{18}$ the valence band offset $\Delta E_{V}^{s / f}$ is given by: 


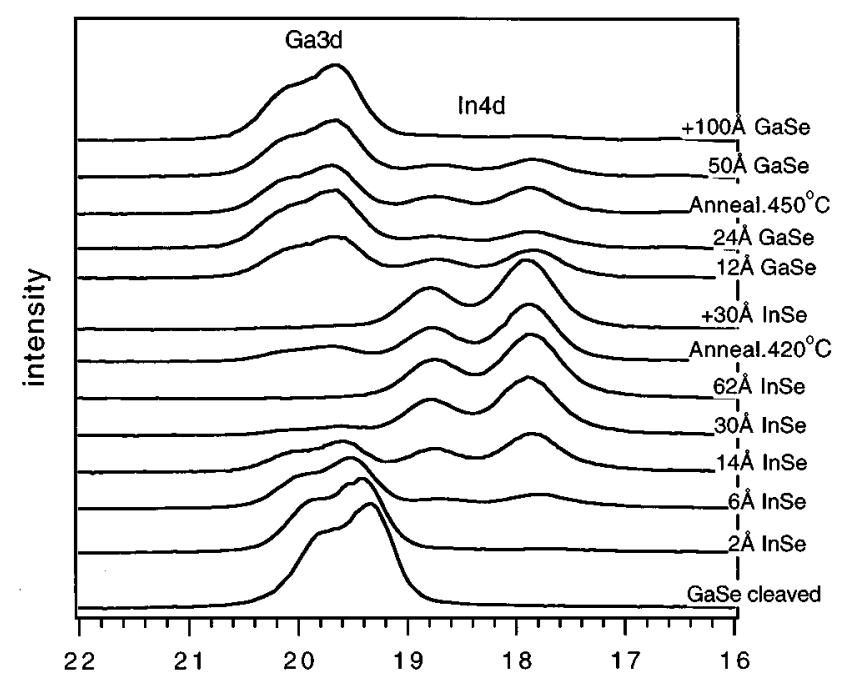

(a)

binding energy $[\mathrm{eV}]$

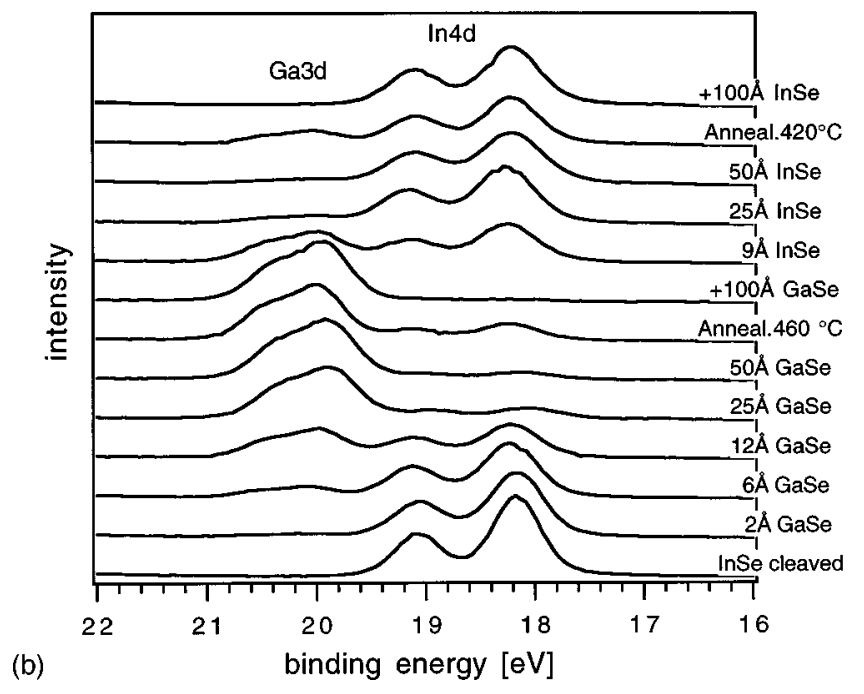

FIG. 2. In part (a) synchrotron excited core level spectra $(h \nu=40 \mathrm{eV})$ of the Ga3d-In4d region are shown in the course of deposition of InSe on GaSe single crystal and subsequent deposition of GaSe. Part (b) shows spectra in the course of deposition of GaSe on InSe single crystal and subsequent deposition of InSe. Intermediate annealing steps are also shown. All spectra were normalized to the incident photon flux. Binding energies are given with respect to the Fermi level.

$$
\begin{aligned}
\Delta E_{V}^{s / f}(\Theta)= & \Delta B E_{\mathrm{CL}}^{s / f}(\Theta)+\left(B E_{\mathrm{CL}}^{s}-B E_{\mathrm{VBM}}^{s}\right) \\
& -\left(B E_{\mathrm{CL}}^{f}-B E_{\mathrm{VBM}}^{f}\right),
\end{aligned}
$$

where the superfixes $s$ and $f$ denote substrate and film, respectively. $B E_{\mathrm{CL}}^{s / f}(\Theta)$ is the binding energy difference of (chemically) unchanged core or valence band emissions of the substrate/film interface for a given coverage $\Theta$.

Core-level binding energies with respect to the VBM $\left(B E_{\mathrm{CL}}^{f / s}-B E_{\mathrm{VBM}}^{f / s}\right)$ are determined from cleaved single crystals. As already mentioned, only small changes in emission angle result in considerable changes of valence band spectra. While the core-level binding energy remains unaffected, we observed shifts of the VBM up to a few hundred meV when rotating the analyzer out off normal emission for $\pm 3^{\circ}$. For an appropriate determination of $B E_{\mathrm{CL}}^{f / s}-B E_{\mathrm{VBM}}^{f / s}$ we therefore
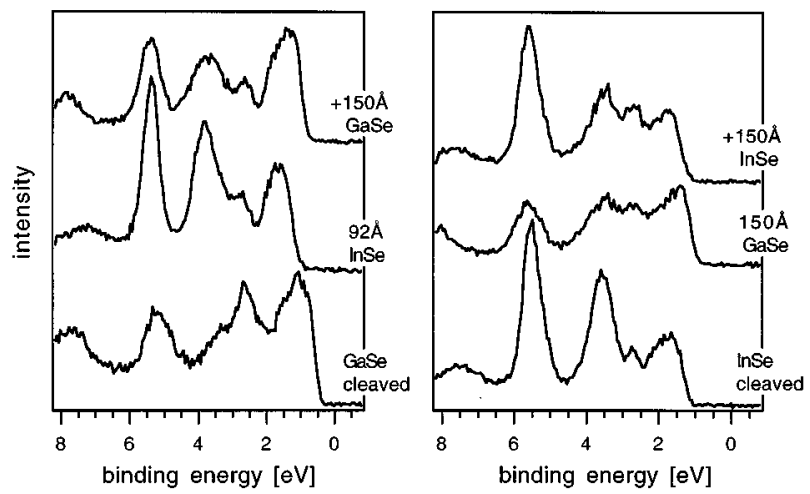

FIG. 3. Synchrotron excited valence band spectra $(h \nu=40 \mathrm{eV})$ for the multilayer systems shown in Figs. 2(a) and 2(b). The spectra are normalized to the incident photon flux.

took valence band spectra with Hell excitation in a Leybold spectrometer EA11 having an acceptance angle of $30^{\circ}$. The procedure is illustrated in Fig. 4. The binding energy of the VBM is determined from extrapolation of the leading edge of the valence band emissions. Core-level binding energies can be determined for InSe from Hell $\alpha(h \nu=40.81 \mathrm{eV})$ and from $\mathrm{Hell}_{\beta}(h \nu=48.37 \mathrm{eV})$ with consistent results. Because of the onset of strong valence band emission from residual Hel intensity and the larger binding energy of the Ga3d level, only the $\mathrm{Hell}_{\beta}$ line can be used in the case of GaSe. Average values for $B E_{\mathrm{CL}}^{f / s}-B E_{\mathrm{VBM}}^{f / s}$ taken from several single crystal surfaces are summarized in Table I.

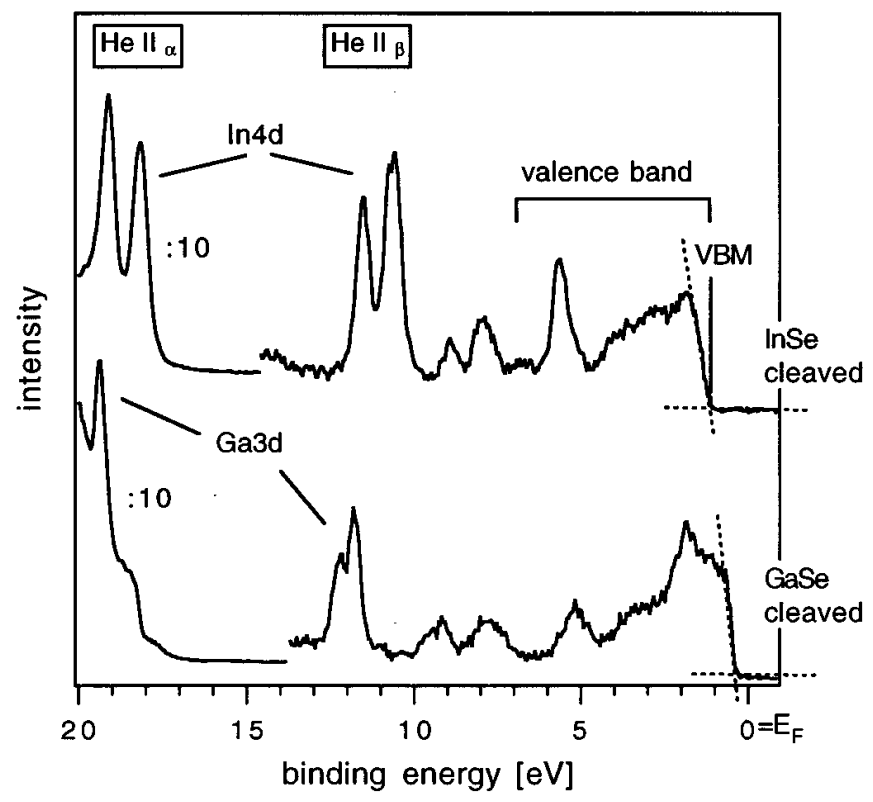

FIG. 4. Hell excited UP-spectra of cleaved InSe and GaSe single crystals as measured with a nonangle resolving spectrometer illustrating the determination of $B E_{\mathrm{CL}}-B E_{\mathrm{VBM}}$ (see text). The valence band maxima $B E_{\mathrm{VBM}}$ are determined from the extrapolation of the leading edges of the valence band emissions. Core level binding energies $B E_{\mathrm{CL}}$ are determined from $\mathrm{Hell}_{\beta}$ excited In4d and Ga3d emissions $(h \nu=48.37 \mathrm{eV})$. In the case of InSe $B E_{\mathrm{CL}}$ may also be determined from the dominating $\mathrm{Hell}_{\alpha}$ excited In $4 \mathrm{~d}$ level. 
TABLE I. Binding energies of core levels $\left(B E_{\mathrm{CL}}\right)$, valence band maximum $\left(B E_{\mathrm{VBM}}\right)$ and work functions $(\varphi)$ as measured with UPS for InSe and GaSe single crystals in units of electronvolt. The values of $B E_{\mathrm{CL}}$ and $B E_{\mathrm{VBM}}$ are with respect to the Fermi energy. The values of $\varphi$ were obtained from the secondary electron cutoff at $h \nu=21.2 \mathrm{eV}$.

\begin{tabular}{lccccc}
\hline \hline$B E_{\mathrm{In} 4 \mathrm{~d} 5 / 2}$ & $B E_{\mathrm{Ga} 3 \mathrm{~d} 5 / 2}$ & $B E_{\mathrm{VBM}}(\mathrm{InSe})$ & $B E_{\mathrm{VBM}}(\mathrm{GaSe})$ & $\varphi(\mathrm{InSe})$ & $\varphi(\mathrm{GaSe})$ \\
\hline 18.25 & 19.38 & 1.1 & 0.35 & 4.7 & 5.3 \\
\hline \hline
\end{tabular}

With these values $\Delta E_{V}^{s / f}$ as determined from binding energy difference $B E(\mathrm{Ga} 3 \mathrm{~d})-B E(\mathrm{In} 4 \mathrm{~d})$ is plotted in Fig. 5 against the coverage $\Theta$ for the $\mathrm{GaSe} / \mathrm{InSe} / \mathrm{GaSe}$ and InSe/GaSe/InSe systems. The graph shows that after an initial variance for low overlayer coverage (below $10 \AA$ ) both systems show $\Delta E_{V}^{s / f}=0.1 \pm 0.05 \mathrm{eV}$ for overlayer thicknesses $\Theta>15 \AA$. This value approximately corresponds to two $X-M-M-X$ layers which form the unit cell of InSe and GaSe crystals. The valence band edge of InSe lies below that of the larger band gap material GaSe, resulting in a so-called staggered lineup.

With the known values of the band gaps (InSe: $E_{G}=1.25$ $\mathrm{eV}$; GaSe: $\left.E_{G}=2.05 \mathrm{eV}\right)^{19}$ the conduction band discontinuities $\Delta E_{C}^{s / f}$ are calculated from:

$$
\Delta E_{C}^{s / f}=\Delta E_{V}^{s / f}-\left(E_{G}^{f}-E_{G}^{s}\right) .
$$

It follows that the conduction minimum of GaSe lies $\Delta E_{C}^{s / f}$ $=0.9 \pm 0.05 \mathrm{eV}$ above that of InSe. The conduction discontinuity $\Delta E_{C}^{s / f}$ can be described in terms of the electron affinities $\chi$ and an interface dipole $\delta$

$$
\Delta E_{C}^{s / f}=\chi^{f}-\chi^{s}+\delta
$$

The electron affinities $\chi=\varphi+\left(E_{f}-B E_{\mathrm{VBM}}\right)$ were again determined by measuring the secondary electron cutoffs and the valence band maxima of UP-spectra with the Leybold

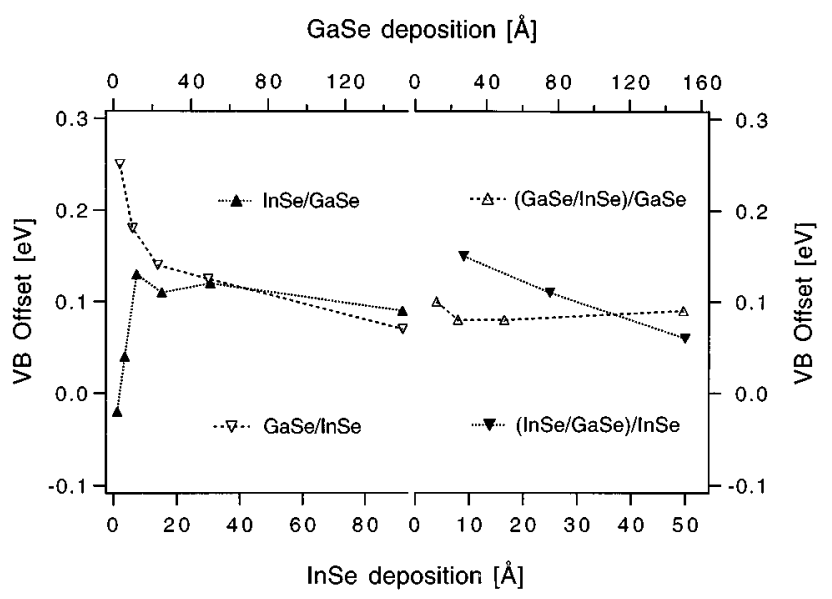

FIG. 5. Valence band off $\Delta E_{V}^{s / f}$ of the InSe/GaSe interfaces determined as described in the text. $\Delta E_{V}^{s / f}$ is plotted against the coverage $\Theta$ for the multilayer systems $\mathrm{GaSe} / \mathrm{InSe} / \mathrm{GaSe}$ (open symbols) and $\mathrm{InSe} / \mathrm{GaSe} / \mathrm{InSe}$ (filled symbols), respectively.

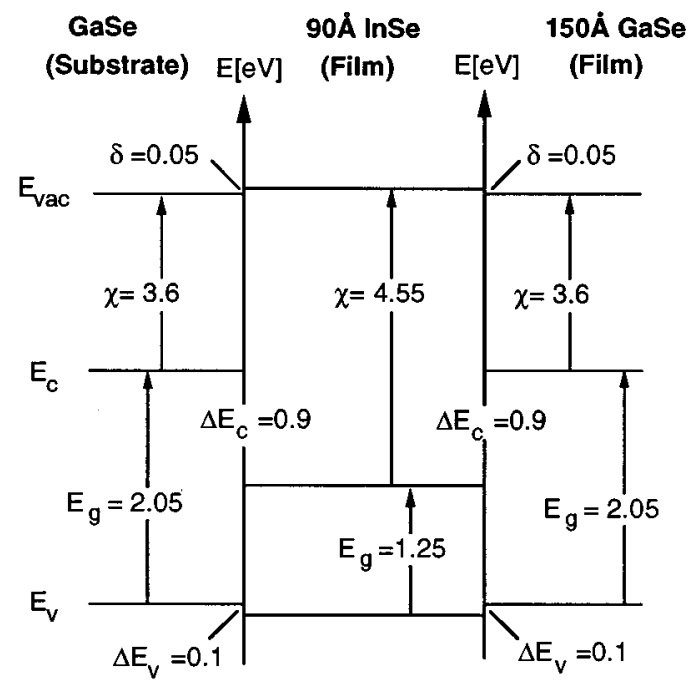

FIG. 6. Schematic energy-band diagram of the GaSe/InSe/GaSe heterosystem as obtained from the presented experiment determination of $\Delta E_{V}$ and $\varphi$ using literature values for the band gaps (Ref. 19). The quantity $\delta$ accounts for a dipole contribution at the interface.

spectrometer for the reasons given above. The Hel-line was used for excitation. The work functions and valence band maxima measured from different InSe and GaSe single crystals exhibit deviations of $\pm 0.1 \mathrm{eV}$. The values given in Table I are averaged between $8 \mathrm{InSe}$ and GaSe single crystals, respectively.

With the above given values for the band gaps and the experimentally determined values for $E_{F}-E_{V}$ and work functions $\varphi$ of cleaved (0001) surfaces of the single crystals, the difference $\delta$ of the vacuum levels across the heterojunction can be evaluated from Eq. (3). The quantity $\delta$ accounts for an additional dipole contribution at the heterointerface and is a measure for the deviation from the $\operatorname{EAR}(\delta=0 \mathrm{eV})$. Our experiment results show that the value of $\delta$ is $0.05 \pm 0.1$ $\mathrm{eV}$ and that the EAR is valid for the van der Waals heterostructures between GaSe and InSe. This result is independent of the deposition sequence (commutativity: $\Delta E_{V}^{A / B}$ $=\Delta E_{V}^{B / A}$ ) as is evident from Figs. 2 and 5. The experimentally determined band diagrams for both structures are summarized in Fig. 6. The validity of the EAR has also been found for the GaSe/WSe 2 VDWE heterointerface. ${ }^{20}$ The small valence band offset of $0.1 \mathrm{eV}$ could have also been expected from the common anion rule. ${ }^{21}$ This would imply that the energetic position of the valence band maxima of InSe and $\mathrm{GaSe}$ is mostly dominated by Se $p$-states.

In summary, epitaxial quantum well structures between the lattice mismatched layered semiconductors InSe and GaSe have been prepared by VDWE. The experimentally determined band alignment is correctly predicted by the electron affinity rule. This observation can be attributed to the ideal structure of the van der Waals surfaces. The chemically saturated hexagonally closed packed selenide layers exist in nearly identical structural and electronic arrangement on the surface as well as in VDWE heterostructures which are evidently nearly identical to the van der Waals gaps within the 
bulk. Therefore, VDWE heterointerfaces are formed without any additional dipole changes which could shift the band edges with respect to each other. As a consequence the electronic properties of the heterointerfaces may be considered as "ideal." They follow the naive rules of band lineup as the EAR (in principle containing surface properties as the surface dipole) as well as the common anion rule (governed by electronic bulk properties). We have also found that commutativity is fulfilled for the band lineups of the investigated quantum well structures.

\section{ACKNOWLEDGMENT}

Technical assistance during measurements by Dipl. Ing. J. Lehmann is greatfully acknowledged. This work was in part supported by a grant of the german ministry of education and research (BMBF) and by EC contract in the Joule II program.

${ }^{1}$ Heterojunction band discontinuities: Physics and device applications, edited by F. Capasso and G. Margaritondo (North-Holland, Amsterdam, 1987).

${ }^{2}$ R. L. Anderson, Solid State Electron. 5, 341 (1962).

${ }^{3}$ J. Tersoff, Phys. Rev. B 30, 4874 (1984).

${ }^{4}$ C. Tejedor and F. Flores, J. Phys. C 12, 731 (1979).

${ }^{5}$ M. Cardona and N. E. Christensen, Phys. Rev. B 35, 6182 (1987).
${ }^{6}$ J. Tersoff, Phys. Rev. Lett. 52, 465 (1984).

${ }^{7}$ See the Proceedings of the 19th and 20th Conference on the Physics and Chemistry of Semiconductor Interfaces [J. Vac. Sci. Technol. B 10, 1674 (1992); ibid. 11, 1329 (1993)].

${ }^{8}$ A. Koma, K. Sunouchi, and T. Miyajima, J. Vac. Sci. Technol. B 3, 724 (1985).

${ }^{9}$ A. Koma, Thin Solid Films 216, 72 (1992).

${ }^{10}$ P. Gomes da Costa, R. G. Dandrea, R. F. Wallis, and M. Balkanski, Phys. Rev. B 48, 14135 (1993).

${ }^{11}$ R. Schlaf, S. Tiefenbacher, O. Lang, C. Pettenkofer, and W. Jaegermann, Surf. Sci. Lett. 303, L343 (1994).

${ }^{12}$ O. Lang, R. Schlaf, Y. Tomm, C. Pettenkofer, and W. Jaegermann, J. Appl. Phys. 75, 7805 (1994).

${ }^{13}$ J. Y. Emery, L. Brahim-Otsmane, C. Hurlimann, and A. Chevy, J. Appl. Phys. 71, 3256 (1991).

${ }^{14}$ N. Nakayama, T. Kuramachi, T. Tanbo, H. Ueba, and C. Tatsuyama, Surf. Sci. 244, 58 (1991).

${ }^{15}$ C. Tatsuyama, T. Tanbo, and N. Nakayama, Appl. Surf. Sci. 41/42, 539 (1989).

${ }^{16}$ A. Chevy, J. Cryst. Growth 67, 119 (1984).

${ }^{17}$ O. Lang, A. Klein, R. Schlaf, T. Löher, C. Pettenkofer, W. Jaegermann, and A. Chevy, J. Cryst. Growth 146, 439 (1995).

${ }^{18}$ J. R. Waldrop and R. W. Grant, Phys. Rev. Lett. 43, 1686 (1979).

${ }^{19}$ E. Bucher, in Photoelectrochemistry and Photovoltaics of Layered Semiconductors, edited by A. Aruchamy (Kluwer Academic, Dordrecht, 1992), p. 1.

${ }^{20}$ O. Lang, Y. Tomm, R. Schlaf, C. Pettenkofer, and W. Jaegermann, J. Appl. Phys. 75, 7814 (1994).

${ }^{21}$ J. O. McCaldin, T. C. McGill, and C. A. Mead, Phys. Rev. Lett. 36, 56 (1976).

This article may be downloaded for personal use only. Any other use requires prior permission of the author and AIP Publishing. This article appeared in Journal of Applied Physics 80, 3817 (1996) and may be found at https://doi.org/10.1063/1.363335.

Available under only the rights of use according to UrhG. 